PROCEEDINGS OF THE

AMERICAN MATHEMATICAL SOCIETY

Volume 125, Number 10, October 1997, Pages 2853-2854

S 0002-9939(97)04042-2

\title{
A CHARACTERIZATION OF CANCELLATION IDEALS
}

\author{
D. D. ANDERSON AND MOSHE ROITMAN
}

(Communicated by Wolmer V. Vasconcelos)

\begin{abstract}
An ideal $I$ of a commutative ring $R$ with identity is called a cancellation ideal if whenever $I B=I C$ for ideals $B$ and $C$ of $R$, then $B=C$. We show that an ideal $I$ is a cancellation ideal if and only if $I$ is locally a regular principal ideal.
\end{abstract}

Let $R$ be a commutative ring with identity. An ideal $I$ of $R$ is called a cancellation ideal if whenever $I B=I C$ for ideals $B$ and $C$ of $R$, then $B=C$. It is easily seen that $I$ is a cancellation ideal if and only if whenever $I B \subseteq I C$ for ideals $B$ and $C$ of $R$, then $B \subseteq C$. A good introduction to cancellation ideals may be found in Gilmer [1, Section 6]. As for examples, it is easy to see that a principal ideal $(a)$ is a cancellation ideal if and only if $(a)$ is a regular ideal (i.e., $a$ is not a zero divisor). An invertible ideal is a cancellation ideal. More generally, an ideal that is locally a regular principal ideal is a cancellation ideal. The purpose of this paper is to prove the converse.

Kaplansky [2, Theorem 287] proved that a finitely generated cancellation ideal in a quasi-local domain is principal. We begin with the following lemma which is a modification of Kaplansky's result (see [1, Exercise 7, page 67]). We use essentially the same argument.

Lemma. Let $R$ be a commutative ring with identity and let $I$ be a cancellation ideal of $R$. Suppose that $I=(x, y)+A$ where $A$ is an ideal of $R$ containing $M I$ for some maximal ideal $M$. Then $I=(x)+A$ or $I=(y)+A$.

Proof. Put $J=\left(x^{2}+y^{2}, x y, x A, y A, A^{2}\right)$. Then it is easily checked that $I J=I^{3}$. Since $I$ is a cancellation ideal, we have $J=I^{2}$. Thus $x^{2}=\lambda\left(x^{2}+y^{2}\right)+$ terms from $\left(x y, x A, y A, A^{2}\right)$. First, suppose that $\lambda \in M$. Since $\lambda x \in M I \subseteq A$, we have $x^{2} \in\left(y^{2}, x y, x A, y A, A^{2}\right)$. Let $K=(y)+A$. Then $I^{2}=I K$. Since $I$ is a cancellation ideal, we have $I=K$. Next, suppose that $\lambda \notin M$. Then for some $\mu \in R$ and $m \in M$, we have $\mu(-\lambda)=1+m$. Now $-\mu \lambda y^{2}=\mu(\lambda-1) x^{2}+$ terms from $\left(x y, x A, y A, A^{2}\right)$. Since $m y^{2}=(m y) y \in(M I) y \subseteq A y$, we have $y^{2} \in\left(x^{2}, x y, x A, y A, A^{2}\right)$. Thus, as in the first case, we get that $I=(x)+A$.

Theorem. Let $R$ be a commutative ring with identity. An ideal $I$ of $R$ is a cancellation ideal if and only if I is locally a regular principal ideal.

Received by the editors May 16, 1996.

1991 Mathematics Subject Classification. Primary 13A15.

Key words and phrases. Cancellation ideal.

M. Roitman thanks the University of Iowa for its hospitality.

(C)1997 American Mathematical Society 
Proof. We have already remarked that an ideal that is locally a regular principal ideal is a cancellation ideal. Conversely, suppose that $I$ is a cancellation ideal. Let $M$ be a maximal ideal of $R$. We show that $I_{M}$ is a regular principal ideal. We may assume that $I \subseteq M$. Choose a subset $\left\{b_{\alpha}\right\}_{\alpha \in \Lambda}$ of $I$ so that $\left\{\bar{b}_{\alpha}\right\}_{\alpha \in \Lambda}$ is a basis for the $R / M$-vector space $I / M I$. Suppose that $|\Lambda|>1$. Then for $\alpha_{1}, \alpha_{2} \in \Lambda$ with $\alpha_{1} \neq \alpha_{2}$, we get $I=\left(b_{\alpha_{1}}, b_{\alpha_{2}}\right)+\left(\left\{b_{\alpha} \mid \alpha \in \Lambda-\left\{\alpha_{1}, \alpha_{2}\right\}\right\}\right)+M I$. By the lemma, say, $I=\left(b_{\alpha_{1}}\right)+\left(\left\{b_{\alpha} \mid \alpha \in \Lambda-\left\{\alpha_{1}, \alpha_{2}\right\}\right\}\right)+M I$. But then $\left\{b_{\alpha} \mid \alpha \in \Lambda-\left\{\alpha_{2}\right\}\right\}$ is a $R / M$-basis for $I / M I$, a contradiction. Hence $I=(a)+M I$ for some $a \in I$. Let $b \in I$. Then $(b) I=(b)((a)+M I)=(a)(b)+M(b) I \subseteq(a) I+M(b) I=$ $((a)+M(b)) I$. Hence $(b) \subseteq(a)+M(b)$. Then $b=r a+m b$ for some $m \in M$, so $(1-m) b=r a$ and hence since $1-m$ is a unit in $R_{M}, b \in(a)_{M}$. Thus $I_{M}=(a)_{M}$. Suppose that $c a=0$ in $R_{M}$. Then $(c I)_{M}=(c a)_{M}=0_{M}$, so $(c I)_{M}=(c M I)_{M}$. Since $(c I)_{N}=(c M I)_{N}$ for all other maximal ideals $N$ of $R$, we have $c I=c M I$. Since $I$ is a cancellation ideal, $(c)=(c) M$. Thus $c=0$ in $R_{M}$. Hence $I_{M}$ is regular.

Corollary 1. Let $R$ be a commutative ring with identity, $S$ a multiplicatively closed subset of $R$, and $I$ a cancellation ideal of $R$. Then $I_{S}$ is a cancellation ideal in $R_{S}$.

We would like to thank the referee for suggesting the following corollary.

Corollary 2. Let $R$ be a subring of the integral domain $T$. If $I$ is a cancellation ideal of $R$, then IT is a cancellation ideal of $T$.

While we have shown that a cancellation ideal $I$ is locally a regular principal ideal, $I$ itself need not be regular. Gilmer [1, Exercise 10, page 456] has given an example of a finitely generated cancellation ideal that is not regular.

\section{REFERENCES}

1. R. Gilmer, Multiplicative ideal theory, Queen's Papers in Pure and Applied Mathematics, vol. 90, Queen's University, Kingston, Ontario, 1992. MR 93j:13001

2. I. Kaplansky, Topics in commutative ring theory, unpublished notes, 1971. MR 55:322

Department of Mathematics, The University of Iowa, Iowa City, Iowa 52242

E-mail address: dan-anderson@uiowa.edu

Department of Mathematics, University of Haifa, Mount Carmel, Haifa 31905, Israel

E-mail address: mroitman@mathcs2.haifa.ac.il 